\title{
Floquet Theory in Electron-Helium Scattering in a Nd:YAG Laser Field
}

\author{
Abdelkader Makhoute ${ }^{1,2^{*}}$, Hicham Agueny ${ }^{1,3}$, Soumia Chqondi ${ }^{1,3}$ \\ ${ }^{1}$ UFR de Physique du Rayonnement et des Interactions Laser-Matière, Faculté des Sciences, \\ Université Moulay Ismail, Meknès, Morocco \\ ${ }^{2}$ The Abdus Salam International Centre for Theoretical Physics, Trieste, Italy \\ ${ }^{3}$ Laboratoire de Chimie Physique-Matière et Rayonnement, Université Pierre et Marie Curie, Paris, France \\ Email: *makhoute@netcourrier.com
}

Received June 27, 2013; revised July 28, 2013; accepted August 25, 2013

Copyright (C) 2013 Abdelkader Makhoute et al. This is an open access article distributed under the Creative Commons Attribution License, which permits unrestricted use, distribution, and reproduction in any medium, provided the original work is properly cited.

\begin{abstract}
The dynamics of laser-assisted elastic collisions in helium is studied. The formalism which will be developed to describe such laser-assisted collisions, treats the laser-projectile interaction to all orders, while the electron-helium interaction is treated within the first Born-approximation. Detailed calculations are performed for the elastic scattering of 50 $\mathrm{eV}$ electrons by helium accompanied by the transfer of photons. The numerical results show that the good physical interpretation of relevant processes needs to consider the maximum of atomic states.
\end{abstract}

Keywords: Laser-Assisted; Elastic Collisions; Born-Approximation; Floquet Theory; Multiphoton Transitions; Low-Lying; High-Lying; Differential Cross Section

\section{Introduction}

Atomic matter exposed to strong radiation fields has become both experimentally and theoretically a broad field of current research. Experimentally, the development of intense and tunable lasers has made possible the observation of multiphoton processes at relatively moderate laser field intensities. The possibility of observing laser-assisted electron impact atomic excitation in the presence of a strong field $[1,2]$, is much more difficult to evidence. In the laser-assisted collisions, dubbed as "Simultaneous Electron-Photon Excitation" (SEPE), the electron-target system can absorb (emit) one or several photons from the laser field, the target atom ending in an excited state. So far, only helium target system and a low frequency field have been considered experimentally: 1) SEPE, in which relatively slow electrons, with incoming energy below the excitation threshold of the metastable $2^{3} \mathrm{~S}$ state, collide with atoms in their ground state $1^{1} \mathrm{~S}$, the laser supplying the needed energy to achieve excitation [REF], 2) SEPE from higher excited states has been also observed [3]. These results raise several questions related to theoretical representation of this class of processes.

The negligeable role of the laser-atom interaction has

"Corresponding author. been investigated by several experiments carried out with low laser field but under conditions where the KrollWatson Approximation (KWA) predicts the vanishing of the free-free cross sections. A very recent study [4] compared KWA results with a R-matrix Floquet calculation of the free-free cross section for $22 \mathrm{eV}$ electrons scattered from helium through angles from $20^{\circ}$ to $70^{\circ}$ in a $\mathrm{CO}_{2}$ laser field. The two calculations were both in excellent agreement with experimental data [4]. Most recently, the results of free-free experiments on electron-helium scattering in a Nd:YAG laser field [5] are perfectly consistent with the calculations using the KWA for large scattering angle.

It is known that the laser-assisted electron-atom collisions can be very sensitive to the dressing of the target by the external radiation field [6,7]. There, the formalism which was developed to describe such laser-assisted collisions treated the laser-projectile interaction to all orders, while the laser-target interaction was treated by using first-order time-dependent perturbation theory. This approach is justified for fast incident electrons and when the electric field strength $\varepsilon_{0}$ remains much weaber than the atomic unit of field strength, $\varepsilon_{0} \ll 5 \times 10^{9} \mathrm{~V} \cdot \mathrm{cm}^{-1}$ and/or when the laser photon energy is far from being resonant with an atomic transition energy $[8,9]$. 
In this paper we describe calculations of differential cross sections for laser-assisted electron-helium collisions in which the interaction between the laser field and the projectile is treated exactly in a non-perturbative way, by using a Volkov wave function [10]. While, the laser-target interaction is treated using the Floquet approach $[9,11]$ for the dominant intermediate state, and perturbatively the remaining target states. Since we are considering fast incident electrons, the interaction between the projectile electron and the target atom is treated using the first Born approximation, and exchange effects are safely neglected [12]. It should be noted that our approach is much less demanding in terms of computational power than the R-Matrix Floquet treatments [4].

In Section 2, we present the theory. In Section 3, laser modifed cross sections and their dependence on laser parameters are discussed, and Section 4 concludes the paper. Atomic units (au) are used throughout this paper.

\section{Theoretical Foundation}

We consider the collision of fast electrons with atoms in intense laser fields. We assume the laser field to be purly monochromatic, linearly polarized, and spatially homogeneous, thus treated classically and can be written as

$$
\boldsymbol{\varepsilon}(t)=\varepsilon_{0} \varepsilon \sin \omega t,
$$

where $\varepsilon_{0}$ is the field amplitude and $\varepsilon$ is its polarization vector. The corresponding vector potential is $\boldsymbol{A}(t)=A_{0} \varepsilon \cos \omega t$, with $A_{0}=c \varepsilon_{0} / \omega$. The wave function of a "free electron interacting with such a field", is given in the velocity gauge and dipole approximation by the Volkov function

$$
\begin{aligned}
& \chi_{k}\left(\boldsymbol{r}_{0}, t\right) \\
& =(2 \pi)^{-3 / 2} \exp \left\{i\left(\boldsymbol{k} \cdot \boldsymbol{r}_{0}-E_{k} t-\alpha_{0} \boldsymbol{\varepsilon} \cdot \boldsymbol{k} \sin \omega t\right)\right\},
\end{aligned}
$$

where $\boldsymbol{r}_{0}$ is the electron coordinate, $\boldsymbol{k}$ denotes the its wave vector, $E_{k}=k^{2} / 2$ is its kinetic energy and $\alpha_{0}=\varepsilon_{0} / \omega^{2}$.

Our main task consists in obtaining an expression for the "dressed" wave function of the initial and final atomic target states in the laser field, valid to all orders interaction. Therefore, we should solve the time-dependent Schrödinger Equation

$$
i \frac{\partial}{\partial t} \Phi_{n}(\boldsymbol{X}, t)=\left[H_{0}+H_{A-F}(t)\right] \Phi_{n}(\boldsymbol{X}, t),
$$

where $\boldsymbol{X}$ denotes the ensemble of the target electrons coordinates, $H_{0}$ is the target atom Hamiltonian in the absence of the external field and $H_{A-L}$ is the atom-field interaction Operator which reads, in the velocity gauge,

$$
H_{A-F}(t)=-\frac{i}{c} \sum_{k=1}^{Z} A(t) \cdot \nabla_{r k}
$$

where $Z$ is the atomic number of the target and $r_{k}$ is the position vector of the $k$ th target electron.

Solving exactly Equation (3) would be a formidable task, but yet hardly achievable.

Therefore, we introduce orthogonal projection operators $P, Q$ such that

$$
P^{2}=P, Q^{2}=Q, P+Q=1, P Q=Q P=0,
$$

where $P$ projects onto the subspace $\mathcal{H}_{P}$ of the states which will be included exactly in the calculation. Note that this subspace should contain the initial and final states of the collision at least.

The Schrödinger Equation (3) is then approximated, in a first stage, by the simplified Equation

$$
i \frac{\partial}{\partial t}\left(P \Phi_{n}\right)=P\left(H_{0}+H_{A-F}\right) P\left(P \Phi_{n}\right),
$$

in which only the few dominant states are coupled. Using the usual Floquet ansatz $[9,13]$ we seek solutions of the form

$$
\begin{aligned}
& P \Phi_{n}(\boldsymbol{X}, t)=\exp \left(-i \frac{\boldsymbol{A} \cdot \boldsymbol{R}}{c}\right) \\
& \cdot \exp \left(-i \varepsilon_{n} t\right) \sum_{m \in \mathcal{H}_{P}} \sum_{N=-\infty}^{+\infty} C_{m n}^{N} \exp (-i N \omega t) \psi_{m}(\boldsymbol{X}),
\end{aligned}
$$

where we have defined $\boldsymbol{R}=\sum_{k=1}^{Z} \boldsymbol{r}_{k}$, and $\psi_{m}$ is a target state of energy $E_{m}$ in the absence of the external field. The Floquet coefficients $C_{m n}^{N}$ and the pseudo-energies $\varepsilon_{n}$ can be found by solving numerically the eigenvalue problem

$$
\begin{aligned}
& \left(E_{m}+N \omega\right) C_{m n}^{N}+\frac{i}{2} \sum_{m^{\prime} \in \mathcal{H}_{P}} M_{m m^{\prime}}\left(C_{m^{\prime} n}^{N-1}-C_{m^{\prime} n}^{N+1}\right) \\
& =\varepsilon C_{m n}^{N},
\end{aligned}
$$

where we have introduced the dipole-coupling matrix elements

$$
M_{m m^{\prime}}=M_{m^{\prime} m}^{*}=\varepsilon_{0}\left\langle\psi_{m}|\boldsymbol{\varepsilon} \cdot \boldsymbol{R}| \psi_{m^{\prime}}\right\rangle .
$$

Finally, a first-order correction to the approximate wave function $P \Phi_{n}$ can be found by treating perturbatively the coupling to all the states which are not included in the subspace $\mathcal{H}_{P}$ (the states of $\mathcal{H}_{Q}$ ) and "switching on" the interaction in an adiabatic way. We obtain

$$
\Phi_{n}=P \Phi_{n}+Q \Phi_{n},
$$

where the first-order approximation to $Q \Phi_{n}$ is a solution of the Equation

$$
i \frac{\partial}{\partial t}\left(Q \Phi_{n}\right)=Q\left(H_{0}+H_{A-F}\right) P\left(P \Phi_{n}\right) .
$$

Explicitly, we have 


$$
Q \Phi_{n}(\boldsymbol{X}, t)=\frac{i}{2} \exp \left(-i \frac{\boldsymbol{A} \cdot \boldsymbol{R}}{c}\right) \exp \left(-i \varepsilon_{n} t\right) \times \sum_{m \in \mathcal{H}_{P}} \sum_{m^{\prime} \in \mathcal{H}_{Q}} \sum_{N=-\infty}^{+\infty} M_{m^{\prime} m} \frac{\left(C_{m n}^{N+1}-C_{m n}^{N-1}\right)}{E_{m^{\prime}}-\varepsilon_{n}-N \omega} \exp (-i N \omega t) \psi_{m^{\prime}}(\boldsymbol{X})
$$

In the last Equation the summation over $m^{\prime}$, where $m^{\prime} \in \mathcal{H}_{Q}$ includes an integration over the continuum states. We remark that the solution (12) is valid for any laser frequencies values, therefore the laser photon energy could be close to the energy of an atomic transition between the initial or final states and an intermediate state of the collision process. In what follows we will denote by $\Phi_{i}$ and $\Phi_{f}$ the dressed initial and final states, respectively.
It should be noted that since the Floquet pseudoenergies $\varepsilon_{n}$ are only defined modulo the photon energy, the Floquet pseudostates are not unique. Our convention will be to define $\Phi_{f}$ as the pseudostate whose pseudoenergie $\varepsilon_{f}$ tends to the unperturbed eigenenergie $E_{f}$ in the limit $\varepsilon_{0} \rightarrow 0$.

The $S$-matrix element for elastic scattering from the ground state, in the direct channel, in the presence of the laser field and in first Born approximation is then given by

$$
S_{e l}^{B_{1}}=-i \int_{-\infty}^{+\infty} \mathrm{d} t\left\langle\chi_{\boldsymbol{k}_{f}}\left(\boldsymbol{r}_{0}, t\right) \Phi_{i}(\boldsymbol{X}, t)\left|V_{d}\left(\boldsymbol{r}_{0}, \boldsymbol{X}\right)\right| \chi_{\boldsymbol{k}_{i}}\left(\boldsymbol{r}_{0}, t\right) \Phi_{i}(\boldsymbol{X}, t)\right\rangle,
$$

where

$$
V_{d}\left(\boldsymbol{r}_{0}, \boldsymbol{X}\right)=-\frac{Z}{r_{0}}+\sum_{j=1}^{Z} \frac{1}{r_{0 j}}
$$

is the electron-target interaction operator.

The time integration is readily performed and leads to an energy-conservation delta function.

$$
S_{e l}^{B_{1}}=i(2 \pi)^{-1} \sum_{\ell=-\infty}^{+\infty} \delta\left(E_{\boldsymbol{k}_{f}}-E_{\boldsymbol{k}_{i}}-\ell \omega\right) f_{e l}^{B_{1}, \ell}(\boldsymbol{K}),
$$

where $f_{e l}^{B_{1} \ell}$, the first Born approximation to the scattering amplitude with the transfer of $\ell$ photons can be written as

$$
f_{e l}^{B_{1}, \ell}(\boldsymbol{K})=f_{1}(\boldsymbol{K})+f_{2}(\boldsymbol{K})+f_{3}(\boldsymbol{K})
$$

with

$$
\begin{aligned}
& f_{1}(\boldsymbol{K})=\sum_{k, k^{\prime}}(i)^{\ell+k-k^{\prime}} J_{\ell+k-k^{\prime}}(\lambda) \times \sum_{n \in \mathcal{H}_{p}} \sum_{m \in \mathcal{H}_{p}} C_{n, 0}^{k^{\prime}, *} C_{m, 0}^{k} f_{m, n}^{B_{1}}(\boldsymbol{K}), \\
& f_{2}(\boldsymbol{K})=\sum_{k, k^{\prime}}(i)^{\ell+k-k^{\prime}} J_{\ell+k-k^{\prime}}(\lambda) \sum_{n^{\prime} \in \mathcal{H}_{Q}} \sum_{n, m \in \mathcal{H}_{p}} M_{n^{\prime}, m} C_{n, 0}^{k^{\prime}, *}\left[\frac{C_{m, 0}^{k-1}+C_{m, 0}^{k+1}}{2\left(\mathrm{E}_{n^{\prime}}-\varepsilon_{0}+k \omega\right)}\right] f_{n, n^{\prime}}^{B_{1}}(\boldsymbol{K}),
\end{aligned}
$$

and

$$
f_{3}(\boldsymbol{K})=\sum_{k, k^{\prime}}(i)^{\ell+k-k^{\prime}} J_{\ell+k-k^{\prime}}(\lambda) \sum_{n^{\prime} \in \mathcal{H}_{Q}} \sum_{n, m \in \mathcal{H}_{p}} M_{n^{\prime}, m}^{*} C_{n, 0}^{k}\left[\frac{C_{m, 0}^{k-1, *}+C_{m, 0}^{k+1, *}}{2\left(\mathrm{E}_{n^{\prime}}-\varepsilon_{0}+k \omega\right)}\right] f_{n^{\prime}, n}^{B_{1}}(\boldsymbol{K}),
$$

In this formula $\boldsymbol{K}=\boldsymbol{k}_{i}-\boldsymbol{k}_{f}$ is the momentum transfer, $J_{\ell+k-k^{\prime}}$ is an ordinary Bessel function of order $\ell+k-k^{\prime}, \quad \lambda=\alpha_{0} \varepsilon \cdot \boldsymbol{K}$ and we have defined the matrix elements,

$$
f_{m, n}^{B_{1}}(\boldsymbol{K})=-\frac{2}{K^{2}}\left\langle\psi_{m}(\boldsymbol{X})\left|\tilde{\mathrm{V}}_{d}(\boldsymbol{K}, \boldsymbol{X})\right| \psi_{n}(\boldsymbol{X})\right\rangle,
$$

This quantity represents the first Born amplitudes corresponding to the scattering event $n \rightarrow m$, in the absence of the external field.

The operator $\tilde{\mathrm{V}}_{d}$ is given by

$$
\tilde{\mathrm{V}}_{d}(\boldsymbol{K}, \boldsymbol{X})=\sum_{j=1}^{Z} \exp \left(i \boldsymbol{K} \cdot \boldsymbol{r}_{j}\right)-Z
$$

using the well known Bethe formula [14]

$$
\int \exp \left(i \boldsymbol{K} \cdot \boldsymbol{r}_{0}\right) \mathrm{V}_{d}\left(\boldsymbol{r}_{0}, \boldsymbol{X}\right) \mathrm{d} \boldsymbol{r}_{0}=\frac{4 \pi}{K^{2}} \tilde{\mathrm{V}}_{d}(\boldsymbol{K}, \boldsymbol{X}),
$$

The first Born differential cross section corresponding to the various multiphoton processes is given by

$$
\left(\frac{\mathrm{d} \sigma^{B_{1}, \ell}}{\mathrm{d} \Omega}\right)_{e l}=\frac{k_{f}(\ell)}{k_{i}}\left|f^{B_{1}, \ell}(\boldsymbol{K})\right|^{2} .
$$

In Equation (16), the first term $f_{1}$, corresponds to the interaction of the laser field with the atomic system in the subspace $\mathrm{H}_{P}$. By retaining only this term, we find that the first Born differential cross section corresponding to the elastic scattering, which is nonperturbative in the subspace $\mathcal{H}_{P}$. with the transfer of $\ell$ photons is given by

$$
\left(\frac{\mathrm{d} \sigma^{B_{1}, \ell}}{\mathrm{d} \Omega}\right)_{e l}=\frac{k_{f}(\ell)}{k_{i}}\left|f_{1}(\boldsymbol{K})\right|^{2}
$$

The amplitudes $f_{2}$ and $f_{3}$ contain infinite sums running over the whole atomic spectrum, and the complement of 
the $H_{P}$ states by those $H_{Q}$ with the transfer of $\ell$ laser photons. These amplitudes have been accurately computed with the help of time-dependent perturbation theory.

An exact evaluation of Equations (18) and (19) is not possible since no general, accurate wave functions are known for all excited states of helium. On the other hand, although the closure approximation could be used to evaluate the terms contained in the summation over $n^{\prime}$, when $n^{\prime}$ is in the subspace $\mathcal{H}_{P}$. We can approximate the "exact" first Born scattering amplitude given by (18) and (19) by including exactly the intermediate states which contribute significantly to the sum, while using the closure approximation to account for the other states. This yields the following approximation for the scattering amplitudes

$$
\begin{aligned}
f_{2}(\boldsymbol{K})= & \sum_{k, k^{\prime}}(i)^{\ell+k-k^{\prime}} J_{\ell+k-k^{\prime}}(\lambda) \sum_{n, m \in \mathcal{H}_{P}} C_{n, 0}^{k^{\prime}, *}\left[C_{m, 0}^{k-1}+C_{m, 0}^{k+1}\right] \\
& \times \varepsilon_{0}\left\{\frac{\left\langle\psi_{n}(\boldsymbol{X})\left|(2 \pi)^{-1} \boldsymbol{\varepsilon} \cdot \boldsymbol{R} \mathrm{e}^{i k \cdot r_{0}} V_{d}(\boldsymbol{K}, \boldsymbol{X})\right| \psi_{m}(\boldsymbol{X})\right\rangle}{2\left(\bar{\omega}_{0}-D_{0}+k \omega\right)}-\sum_{n^{\prime} \in \mathcal{H}_{P}} \frac{M_{n^{\prime}, m}^{*}}{2\left(E_{n^{\prime}}-\varepsilon+k \omega\right)}\right\} f_{n, n^{\prime}}^{B_{1}}(\boldsymbol{K})
\end{aligned}
$$

and

$$
\begin{aligned}
& f_{3}(\boldsymbol{K})=\sum_{k, k^{\prime}}(i)^{\ell+k-k^{\prime}} J_{\ell+k-k^{\prime}}(\lambda) \sum_{n, m \in \mathcal{H}_{P}} C_{n, 0}^{k^{\prime}}\left[C_{m, 0}^{k-1}+C_{m, 0}^{k+1}\right] \\
& \times \varepsilon_{0}\left\{\frac{\left\langle\psi_{m}(\boldsymbol{X})\left|(2 \pi)^{-1} \boldsymbol{\varepsilon} \cdot \boldsymbol{R} \mathrm{e}^{i k \cdot r_{0}} V_{d}(\boldsymbol{K}, \boldsymbol{X})\right| \psi_{n}(\boldsymbol{X})\right\rangle}{2\left(\bar{\omega}_{0}-D_{0}+k^{\prime} \omega\right)}-\sum_{n^{\prime} \in \mathcal{H}_{P}} \frac{M_{n^{\prime}, m}}{2\left(E_{n^{\prime}}-\varepsilon+k^{\prime} \omega\right)}\right\} f_{n, n^{\prime}}^{B_{1}}(\boldsymbol{K}),
\end{aligned}
$$

where $D_{0}=\varepsilon_{0}-E_{0}, \bar{\omega}_{0}$ is the average difference between the energy of intermediate state that of the ground state (i.e. the average excitation energy). For $\bar{\omega}_{0}$ we have chosen the value 1.15 a.u., which gives the correct dipole polarizability of the helium ground, state $\bar{\alpha}=1.38$. In writing down Equations (25) and (26), we have only considered the case of final and intermediate states in the subspace $\mathcal{H}_{P}$, since exchange effects (which are small at high energies) are not included in our treatment. The sum over $n^{\prime}$ appearing in Equations (25) and (26) now involves only those intermediate states which we treat exactly.

For the ground state we use the wave function [9]

$$
\psi_{0}\left(\boldsymbol{r}_{1}, \boldsymbol{r}_{2}\right)=\Phi_{0}\left(\boldsymbol{r}_{1}\right) \Phi_{0}\left(\boldsymbol{r}_{2}\right),
$$

where the orbital $\Phi_{0}(\boldsymbol{r})$ is given by

$$
\Phi_{0}(\boldsymbol{r})=\frac{1}{4 \pi} R_{0}^{H e}(r)=\frac{1}{4 \pi}\left(A \mathrm{e}^{-\alpha r}+B \mathrm{e}^{\beta r}\right),
$$

with $A=2.60505, B=2.08144, \alpha=1.41$ and $\beta=2.61$. For the $2^{1} S$ state we have chosen the wave function $[9,15]$

$$
\psi_{2^{1} S}\left(\boldsymbol{r}_{1}, \boldsymbol{r}_{2}\right)=C\left[u_{1}\left(\boldsymbol{r}_{1}\right) u_{2}\left(\boldsymbol{r}_{2}\right)+u_{1}\left(\boldsymbol{r}_{2}\right) u_{2}\left(\boldsymbol{r}_{1}\right)\right],
$$

where

$$
u_{1}(\boldsymbol{r})=\frac{1}{\sqrt{4 \pi}} C_{1} \mathrm{e}^{-2 r}
$$

and

$$
u_{2}(\boldsymbol{r})=\frac{1}{\sqrt{4 \pi}} C_{2}\left(\mathrm{e}^{-\tau_{1} r}-S r \mathrm{e}^{-\tau_{2} r}\right) .
$$

The values of the parameters being $C=0.705226, C_{1}=$ 5.656854, $C_{2}=0.619280, \tau_{1}=0.865, \tau_{2}=0.522$ and $S=$ 0.432785

The intermediate states $2^{1} P$ and $3{ }^{1} P$, are represented by expressions of the form

$$
\begin{aligned}
\psi_{n^{1} P}\left(\boldsymbol{r}_{1}, \boldsymbol{r}_{2}\right)= & \frac{1}{\sqrt{2}}\left[\psi_{1 S}\left(Z_{i}, \boldsymbol{r}_{1}\right) \psi_{n p m}\left(Z_{0}, \boldsymbol{r}_{2}\right)\right. \\
& \left.+\psi_{1 S}\left(Z_{i}, \boldsymbol{r}_{2}\right) \psi_{n p m}\left(Z_{0}, \boldsymbol{r}_{1}\right)\right],
\end{aligned}
$$

where $\psi_{1 s}$ and $\psi_{n p m}$ are hydrogenic wave function corresponding to $1 s$ and npm states with effective charges $Z_{i}=2$ and $Z_{0}=1$, respectively, and the index $\mathrm{n}$ can be take both the values 2 and. Since we want to include exactly all intermediate states with principal quantum number $n \leq 3$, we also need in the present case the wave functions of the $3{ }^{1} S$ and $3{ }^{1} D$ states. For the $3{ }^{1} S$ state we have used the function $[9,15]$

$$
\psi_{3^{1} S}\left(\boldsymbol{r}_{1}, \boldsymbol{r}_{2}\right)=N\left[u_{1}\left(\boldsymbol{r}_{1}\right) u_{3}\left(\boldsymbol{r}_{2}\right)+u_{1}\left(\boldsymbol{r}_{2}\right) u_{3}\left(\boldsymbol{r}_{1}\right)\right],
$$

where $u_{1}(r)$ is given by (41),

$$
u_{3}(\boldsymbol{r})=\frac{1}{4 \pi} \tilde{N}\left[\mathrm{e}^{-\sigma_{1} r}-\Gamma r \mathrm{e}^{-\sigma_{2} r}+\Lambda r^{2} \mathrm{e}^{-\sigma_{3^{r}}}\right]
$$

and the values of the parameters are $N=0.512410, \tilde{N}$ $=0.456615, \sigma_{1}=0.331, \sigma_{2}=0.464, \sigma_{3}=0.330, \Gamma=$ 0.932435 , and $\Lambda=0.0038820$. This function is orthogonal to the $1^{1} S$ wave function (27) and the $2^{1} S$ wave function (29), and gives the accurate value -2.0606 a.u. for the energy of the $3^{1} S$ state. For the $3^{1} D$ state, we have used a wave function of the type $[9,15]$ 


$$
\begin{aligned}
\psi_{3^{1} D}\left(\boldsymbol{r}_{1}, \boldsymbol{r}_{2}\right)= & \frac{1}{\sqrt{2}}\left[\psi_{1 S}\left(Z_{i}, \boldsymbol{r}_{1}\right) \psi_{3 d m}\left(Z_{0}, \boldsymbol{r}_{2}\right)\right. \\
& \left.+\psi_{1 S}\left(Z_{i}, \boldsymbol{r}_{2}\right) \psi_{3 d m}\left(Z_{0}, \boldsymbol{r}_{1}\right)\right]
\end{aligned}
$$

where $\psi_{1 S}$ and $\psi_{3 d m}$ are hydrogenic wave function corresponding to the effective charges $Z_{i}=2$ and $Z_{0}=1$, respectively.

\section{Results and Discussion}

We will discuss the variation of the cross-sections corresponding to the elastic collisions in terms of the parameters governing the collision dynamics, i.e., the scattering angle and number of photons exchanged during the collision. We will also discuss the influence of the laser polarization on the processes, which plays a purely geometrical role. In particular it can give rise to a strong asymmetry between absorption and emission. Our results refer to an incident electron energy $E_{k_{i}}=50 \mathrm{eV}$, a fixed $\mathrm{Nd}$ :YAG laser photon energy $\hbar \omega=1.17 \mathrm{eV}$ and typical electric field strengths $\varepsilon_{0}=10^{8} \mathrm{~V} \cdot \mathrm{cm}^{-1}$. Moreover, we have considered here three particular geometries where the polarization vector of the field is taken to be parallel to the momentum transfer $\boldsymbol{K}$, or to be parallel to the momentum of the incident electron $\boldsymbol{k}_{i}$ and or to be perpendicular to the incident momentum.

In Figure 1, we display the differential cross section accompanying $\ell=0,+1$ photons exchanged between the electron-atom system and the radiation field. We presente the effects of the states which are not included in the subspace $\mathrm{H}_{P}$ by comparing the results obtained from Equation (23) (in which only the dominant states are coupled) and Equation (24) (which can be found by treating perturbatively the coupling to all the states which are note included in the subspace $\mathrm{H}_{P}$ ) in the same graph for different geometries.

Our results are interpreted by considering the firstBorn differential cross sections, for a fixed electric field strength and a fixed laser photon energy. We have examined our treatment in first Born approximation as a function of the scattering angles and they give similar results beyond $50 \mathrm{eV}$ for the incoming electron energies. For the scattering without any net exchange of photons the differences between the results obtained from Equation (23) and those obtained from Equation (24) are too small to be seen on the scale of Figure 1 and can be neglected. In contrast, for the net exchange of one photon, the differences are very important at small scattering angles and are otherwise constant for a given incident energy and a fixed $\ell$ for different geometries. This is due to the presence the states which are not included in the subspace $\mathrm{H}_{P}$. For a calculation valid to all orders in the interaction between the radiation field and the atomic states of the collision, and for a good physical interpreta- tion of the results requires to consider the maximum atomic states.

In Figure 2 we display the differential cross-section accompanying $\ell=0, \pm 1, \pm 2$ photons exchanged for three distinct geometries for the laser polarization vector $\boldsymbol{\varepsilon}$ either parallel or perpendicular to the incident momentum $\boldsymbol{k}_{\boldsymbol{i}}$ and parallel to momentum transfer $\boldsymbol{K}$.

The results displayed in the set of Figure 2 correspond to the complete results obtained by using the scattering amplitude (16) for three different geometries. The features of these graphs are more or less the same. Each cross-section oscillates by a few orders over the whole scattering angular range. However, there is a significant difference between the results of three geometries in each graph: the cross-section for a parallel geometry oscillate more frequently in the intermediate angles, and its envelop (not drown in each graph) declines with scattering angle increasing; in contrast, the results for a perpendicular geometry oscillate more frequently at the forward and backward angles, and its envelop (not present in each graph) rise after $\theta=90^{\circ}$. While for the case in which the polarization vector $\boldsymbol{\varepsilon}$ of the field is parallel to the momentum transfer $\boldsymbol{K}$, the cross section oscillate more frequently at the forward angles and its envelop declines with scattering angle increasing. The Bessel function $J_{\ell}\left(\alpha_{0} \varepsilon \cdot \boldsymbol{K}\right)$ appearing in the direct amplitudes of Equations (17)-(19) are responsible for the cross-section oscillations. For a parallel polarization geometry, the argument of Bessel unction changes slowly against the scattering angle in the forward and backward direction, which cause the cross-sections oscillating slowly; in medium angular range the argument varies rapidly, and leads to the rapid oscillation of cross-section at mediate angles. For a perpendicular polarization geometry, the situation is opposite: the argument of Bessel functions varies rapidly at small and large angles, but slowly at intermediate angles, thus leads to the opposite feature in the perpendicularly polarized cross-sections. For the geometry where the polarization vector of the field is taken to be parallel to the momentum transfer $\boldsymbol{K}$, the argument of the Bessel function being then reduced to an identical value $K \alpha_{0}$, which varies in the same sense as scattering angle. This causes the cross sections oscillating declines with scattering angle increasing.

In the case of elastic collisions, dressing effects to be dominant in the forward direction for a given laser photon energy. This behavior is illustrated in the set of Figure 3, where we present the differential cross-sections for laser-assisted scattering with the net exchange of one photon $(\ell= \pm 1)$ as a function of the scattering angle $\theta$ and for three distinct polarizations of the field ( $\varepsilon \| \boldsymbol{K}$, $\boldsymbol{\varepsilon} \| \boldsymbol{k}_{i}$ and $\left.\boldsymbol{\varepsilon} \perp \boldsymbol{k}_{i}\right)$. The complete results obtained by using amplitude (24) is compared to the cross section obtained by using first-order time-dependent perturbation 


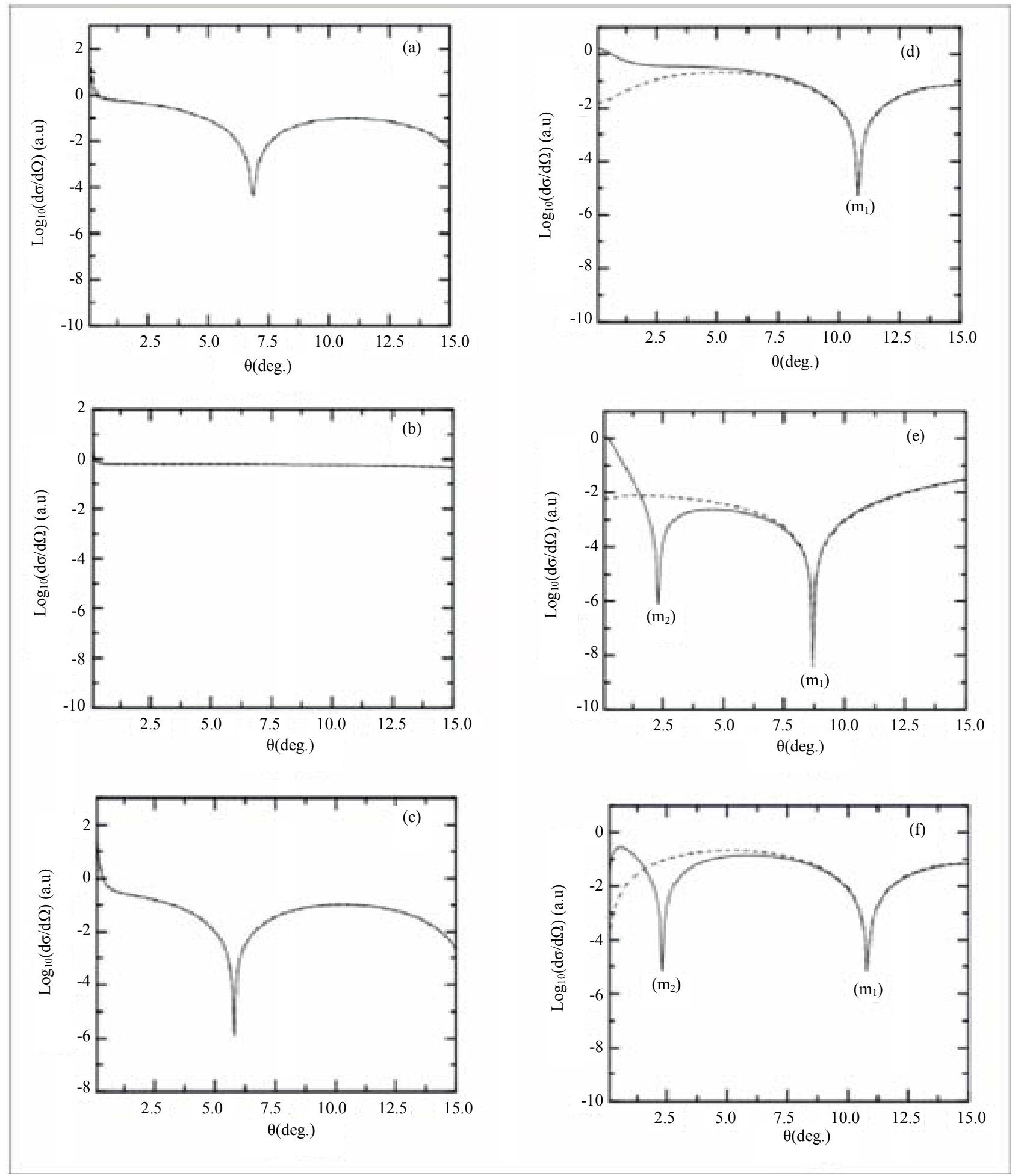

Figure 1. Variation of $\log _{10}(\mathrm{~d} \sigma / \mathrm{d} \Omega)$ as function of the scattering angle $\theta$. The incident electron energy is $E_{k_{i}}=50 \mathrm{eV}$, the laser photon energy is $\hbar \omega=1.17 \mathrm{eV}$ and the electric field strength is $\varepsilon_{0}=10^{8} \mathrm{~V} \cdot \mathrm{cm}^{-1}$. (a) Non photon exchange $(\ell=0)$ with $\varepsilon_{0} \perp K$. (b) Non photon exchange $(\ell=0)$ with $\varepsilon_{0} \| k_{i} \cdot(c)$ Non photon exchange $(\ell=0)$ with $\varepsilon_{0} \| k_{i} \cdot(d)$ One-photon absorption $(\ell=1)$ with $\varepsilon_{0} \| K$. (e) one-photon absorption $(\ell=1)$ with $\varepsilon_{0} \| k_{i} \cdot(f)$ one-photon absorption $(\ell=1)$ with $\varepsilon_{0} \perp k_{i}$. Solid lines gives the nonperturbative results obtained by using Equatio $\mathbf{n}(23)$. Dashed lines give the nonperturbative results obtained by using Equation (24). 


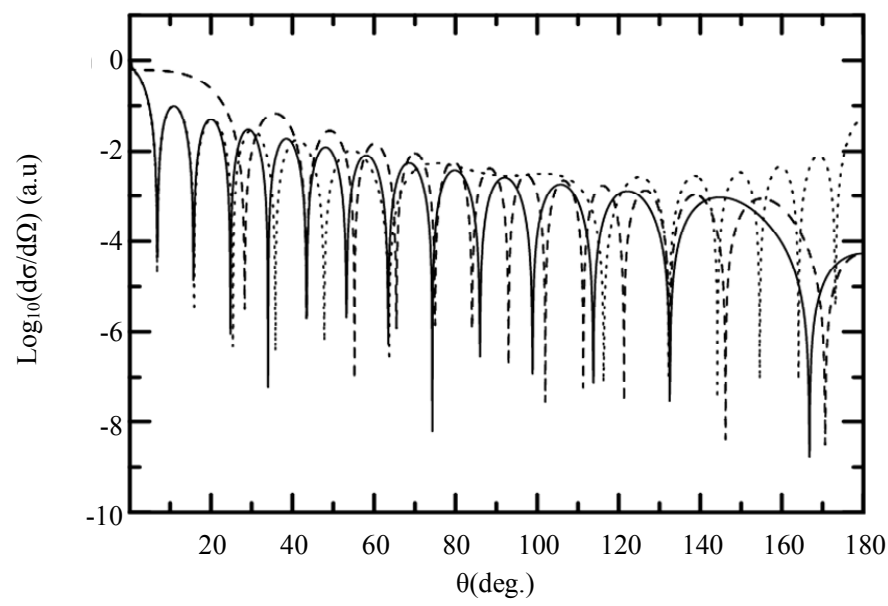

(a)

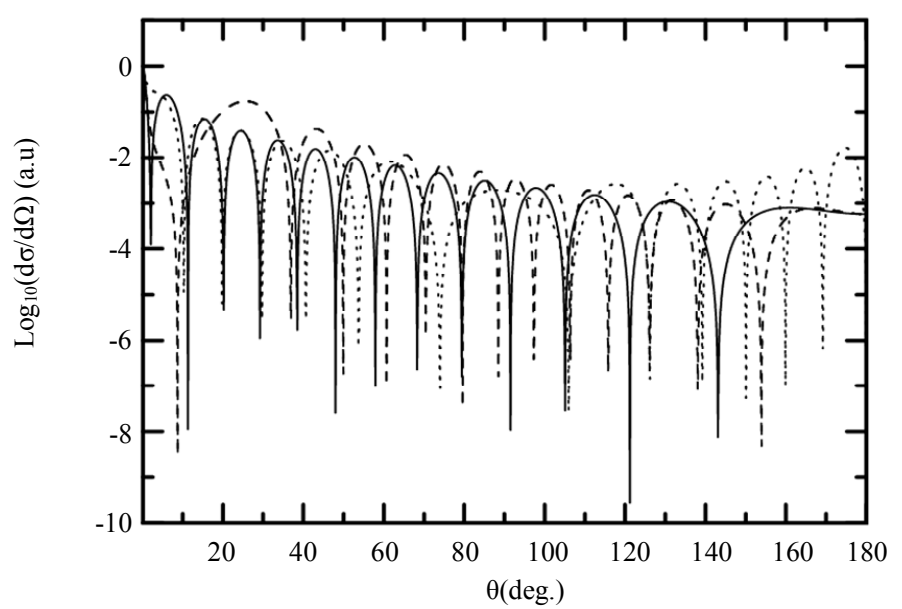

(b)

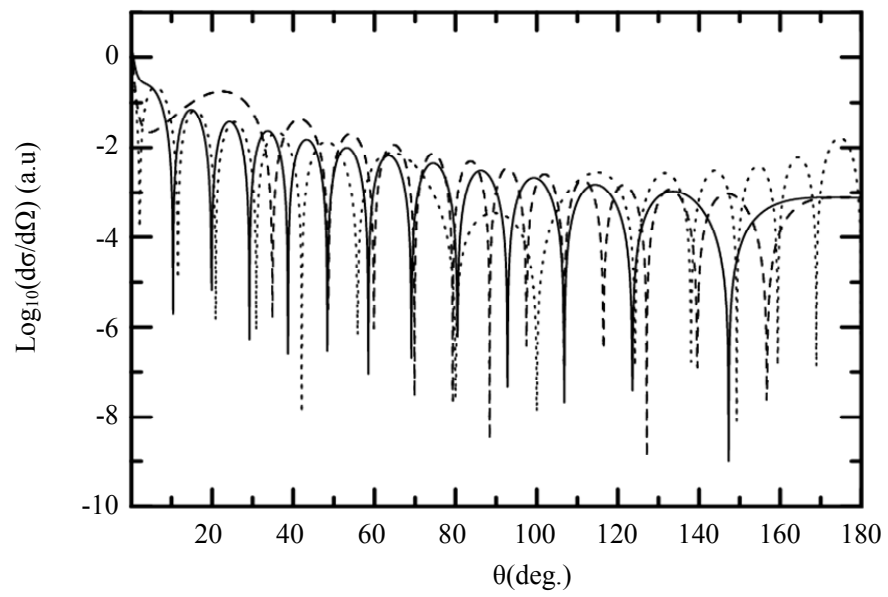

(c)

Figure 2. Variation of $\log _{10}(\mathrm{~d} \sigma / \mathrm{d} \Omega)$ corresponding to the nonperturbative results obtained by using the amplitudes of Equation (35) as function of the scattering angle $\theta$. The incident electron energy is $E_{k_{i}}=50 \mathrm{eV}$, the laser photon energy is $\hbar \omega=1.17 \mathrm{eV}$ and the electric field strength is $\varepsilon_{0}=10^{8} \mathrm{~V} \cdot \mathrm{cm}^{-1}$. (a) Non photon exchange $(\ell=0)$, (b) One-photon absorption $(\ell=1)$, (c) One-photon emission $(\ell=-1)$. Solid lines: The polarization vector $\varepsilon$ taken to be parallel to the momentum transfer direction $K$. Dashed lines: The polarization vector is chosen to be parallel to the incident electron momentum direction $k_{i}$. Dotted lines: The polarization vector is chosen to be perpendicular to the incident electron momentum direction $k_{i}$. 


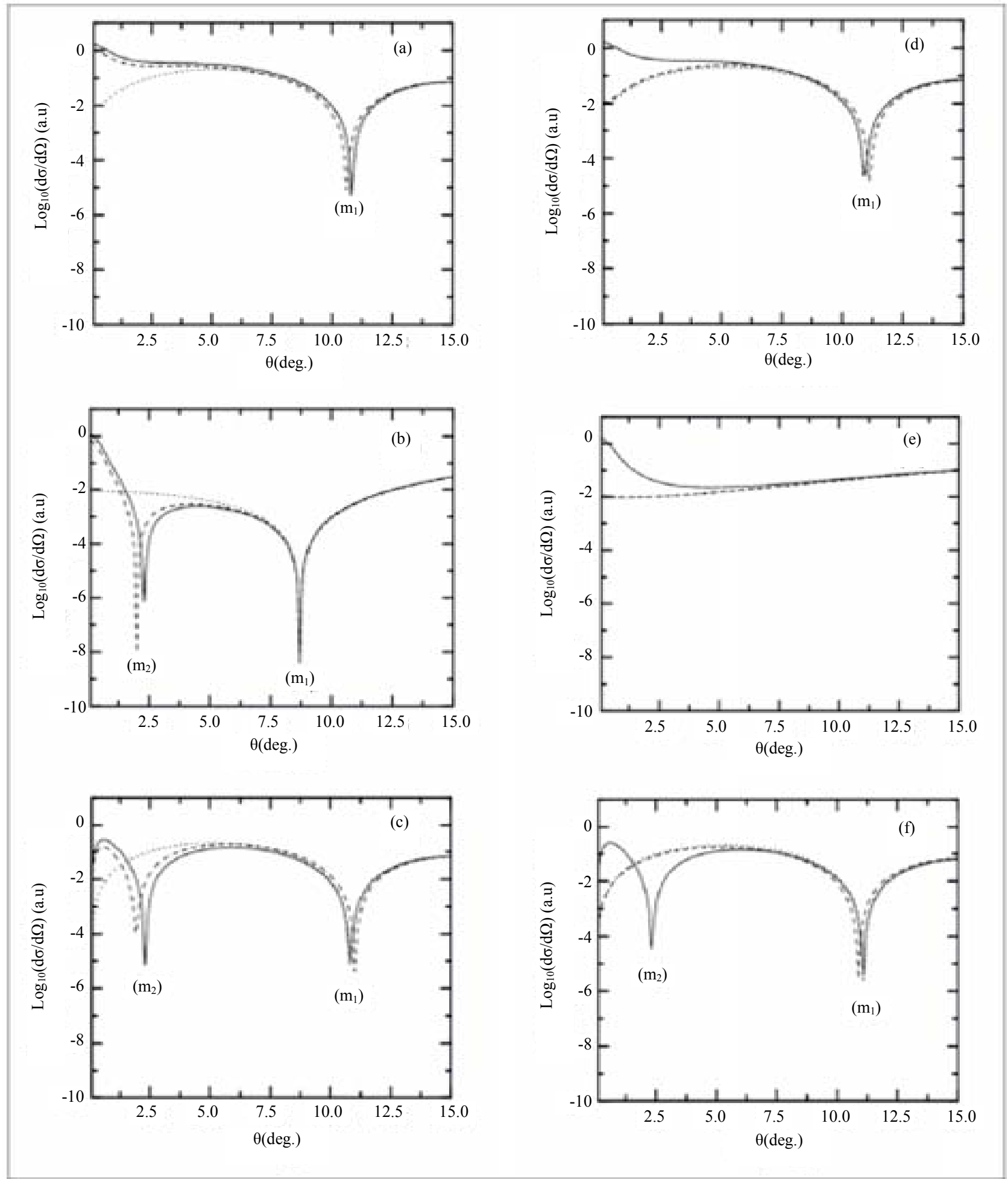

Figure 3. Variation of $\log _{10}(\mathrm{~d} \sigma / \mathrm{d} \Omega)$ as function of the scattering angle $\theta$. The incident electron energy is $E_{k_{i}}=50 \mathrm{eV}$, the laser photon energy is $\hbar \omega=1.17 \mathrm{eV}$ and the electric field strength is $\varepsilon_{0}=10^{8} \mathrm{~V} \cdot \mathrm{cm}^{-1}$. (a) One-photon absorption $(\ell=1)$ with $\varepsilon \| K$. (b) One-photon absorption $(\ell=1)$ with $\varepsilon \| k_{i}$. (c) One-photon absorption $(\ell=1)$ with $\varepsilon \perp k_{i}$. (d) One-photon emission $(\ell=-1)$ with $\varepsilon \| K$. (e) One-photon emission $(\ell=-1)$ with $\varepsilon \| k_{i}$. (f) One-photon emission $(\ell=-1)$ with $\varepsilon \perp k_{i}$. Solid lines: The nonperturbative results obtained by using Equation (24). Dashed lines: The perturbative results. Dotted lines: The results obtained by neglecting the dressing of the target. 
theory and to the "electronic" cross section in which dressing effects are neglected [7].

As indicated in our previous paper on elastic scattering of helium [16] and excitation of atomic hydrogen [17], we have observed the existence of two kinds of minima noted $\left(m_{1}\right)$ and $\left(m_{2}\right)$ on the differential cross sections. Both minima correspond to values of the scattering angle for which the cross section is actually zero; nevertheless the origin of these zeros is different for each case.

The minimum denoted $\left(m_{1}\right)$ occur in fact at angles such that the argument $\lambda$ of the Bessel functions actually vanishes. Those minima appear at the same angle for several curves, i.e. this product is common to the different scattering amplitudes. Moreover, we notice that those minima exist in the localization in $\theta$ is given by the following Equation

$$
k_{i}-k_{f} \cdot \cos \theta=0 .
$$

We note that the condition $\theta=\cos ^{-1}\left(k_{i} / k_{f}\right)$, should be modified in the case of more general geometries corresponding to different orientations of laser polarization [18]. On the other hand the minimum denoted $\left(m_{2}\right)$ appears at angles for which the first Born differential crosssection vanishes, i.e. when $f_{1}(\boldsymbol{K})+f_{2}(\boldsymbol{K})+f_{3}(\boldsymbol{K})=0$. This behavior results from the fact that the resonant atomic amplitudes $f_{2}$ and $f_{3}$ change sign in this range and can compensate the direct contribution $f_{1}$ (the direct and the atomic amplitudes are varying in opposites directions when the momentum transfer increases), which a destructive interferences. This minimum exists for absorption with net exchange of photons in the cases $\boldsymbol{\varepsilon} \| \boldsymbol{k}_{i}$ and $\boldsymbol{\varepsilon} \perp \boldsymbol{k}_{i}$ and only for emission in the case when $\boldsymbol{\varepsilon} \perp \boldsymbol{k}_{i}$. In contrast, for the case of the laser field is chosen to be parallel to the momentum transfer $\boldsymbol{K}$ (see set of the Figure 3), the differential cross-sections display only one minimum designed $\left(m_{1}\right)$ because the condition $\boldsymbol{\varepsilon} \cdot \boldsymbol{K}=0$ can be met at any scattering angle, while the minima $\left(m_{2}\right)$ disappear. For a given incident and photon energies, the absence of the minima $\left(m_{1}\right)$ and/or $\left(m_{2}\right)$ make the difference between emission and absorption, when the polarization vector of the field either parallel or perpendicular to the momentum of the incident electron $\boldsymbol{k}_{i}$. This difference comes from the condition $\boldsymbol{\varepsilon} \cdot \boldsymbol{K}=0$, which cannot be fulfilled in the emission cases $\boldsymbol{\varepsilon} \cdot \boldsymbol{K}$ and $\boldsymbol{\varepsilon} \| \boldsymbol{k}_{i}$ for purely kinematical reasons $\left(\ell \omega=E_{k_{i}} \cdot \operatorname{tg}^{2} \theta\right)$. The last condition was justified when $\boldsymbol{\varepsilon} \perp \boldsymbol{k}_{i}$. The presence of a destructive interference between the direct $f_{1}$ and the atomic amplitudes $f_{2}$ and $f_{3}$ is a general feature of $1{ }^{1} S \rightarrow \mathrm{n}^{1} S$ transitions, in particular for $1{ }^{1} S \rightarrow 1^{1} S$, in the case of absorption $\ell \geq 1$ for $\boldsymbol{\varepsilon} \| \boldsymbol{k}_{i}$ and $\boldsymbol{F}_{0} \perp \boldsymbol{k}_{i}$ and in the case of emission $\ell \leq 1$ for $\boldsymbol{\varepsilon} \perp \boldsymbol{k}_{i}$. This is due to the presence, in the atomic term $\left(f_{2}+f_{3}\right)$ of $\mathrm{s}-p$ transition amplitudes, which behave like $K^{-1}$ for small $K$. This behavior can be explained by change of Bessel functions from absorption $\ell=1,2, \cdots$ to emission $\ell=-1,-2, \cdots$ making a change of sign of the atomic amplitude $\left(f_{2}+f_{3}\right)$. This change is very important in the case when $\boldsymbol{\varepsilon} \| \boldsymbol{k}_{i}$ and $\boldsymbol{\varepsilon} \perp \boldsymbol{k}_{i}$.

In Figure 3 the shape of the differential cross section with the nonperurbative treatment follows the same behaviour that corresponds to the results obtained in the case of the first-order perturbation theory for such a choice for the field amplitude. However, the agreement between the perturbative and present treatment results corresponding to the elastic scattering, with $\ell= \pm 1$, is occurred. Once again, except for small scattering angles with strong laser field, this agreement between both methods is excellent. Finally, this predicts important asymmetries between inverse and stimulated bremsstrahlung. That feature constitutes one of the main differences between elastic and inelastic scattering in a laser field [7].

Figure 4 shows the two-photon to one-photon freefree cross section ratio at $300 \mathrm{eV}$ incident energy as a function of a laser intensity. The increase in the ratio with intensity indicates that a measurement of the ratio at $300 \mathrm{eV}$, made by deHarak et al. [5], can be used to deduce the laser intensity within the validity of the KWA and our treatment at the lowes laser intensity. The neglect of the laser-atom interaction can be carried out with moderate laser power where the KWA predicts very small, or indeed vanishing free-free cross sections under certain circumstances [5].

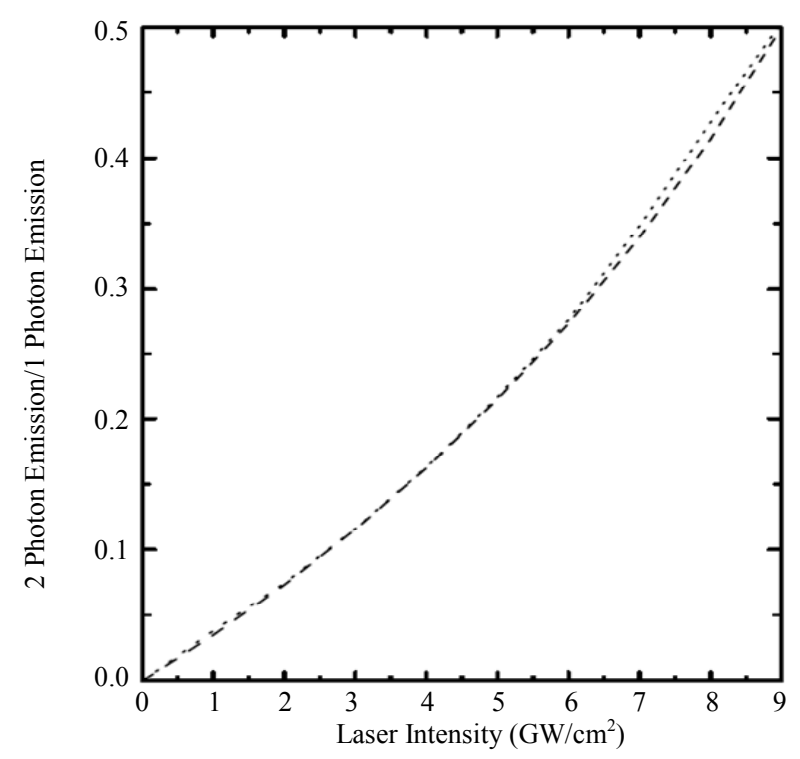

Figure 4. Variation of ratio of two-photon to one-photon emission free-free cross sections as a function of laser intensity for $300 \mathrm{eV}$ incident electrons. The scattering angle is $135^{\circ}$, and the laser polarization is parallel to the scattered electron direction. Dashed line: The nonperturbative results obtained by using Equation (35). Ditted line: The results obtained by deHarak et al. [5]. 


\section{Conclusions}

We have elaborated a treatment of electron-atom collisions in the presence of laser field. This treatment can be applied in the case of a nearly resonant laser field where the perturbation theory diverges. Our method treats to all the interaction of the field with the fast incident projectile, as well as the interaction of the field with the dominant (low-lying) atomic states. This latter interaction is treated by using Floquet theory of multiphoton transitions. Finally, the coupling of the laser field with the high-lying states of the atomic spectrum is treated perturbatively.

We have applied this method to the electron-helium elastic collisions, in the presence of a nonresonant laser field. The very interesting effect is that the numerical results show that during such a laser-assisted elastic collisions, the electron-atom system may exchange a great number of photons with the laser background. Each multiphoton cross sections oscillate by a few orders over the whole scattering angular region. For parallel polarization geometry $\boldsymbol{\varepsilon} \| \boldsymbol{k}_{i}$, the results oscillate more frequently in the medium angular range than in the forward and backward angles; while for a perpendicular geometry $\boldsymbol{\varepsilon} \perp \boldsymbol{k}_{i}$, the oscillation feature is opposite.

Our results have been compared with those obtained by treating the laser-atom interaction by first-order timedependent perturbation theory. The agreement between both methods is good, except close to the resonance (when the laser frequency is close to the Bohr frequency) where the perturbative cross sections diverge [7], while the nonperturbative one exhibits no maximum as a function of the laser frequency and for small scattering angle.

\section{REFERENCES}

[1] N. J. Mason and W. R. Newell, "Simultaneous ElectronPhoton Excitation of the Helium $2^{3}$ S State," Journal of Physics B, Vol. 20, 1987, p. L323.

http://dx.doi.org/10.1088/0022-3700/20/10/006

[2] B. Wallbank, J. K. Holmes, L. Le Blanc and A. Weingartshofer, "Simultaneous Off-Shell Excitation of He $2^{3} \mathrm{~S}$ by an Electron and One or More Photons," Zeitschrift für Physik D, Vol. 10, 1988, p. 467.

[3] B. Wallbank, J. K. Holmes and A. Weingartshofer, "Simultaneous Electron-Photon Ex-Citation of $\mathrm{He} 2^{3} \mathrm{~S}$ : An Experimental Investigation of the Effects of Laser Intensity and Polarisation," Journal of Physics B, Vol. 23, 1990, p. 2997. http://dx.doi.org/10.1088/0953-4075/23/17/012

[4] D. Nehari, J. Holmes, K. M. Dunseath and M. TeraoDunseath, "Experimental and Theoretical Study of FreeFree Electron-Helium Scattering in a $\mathrm{CO}_{2}$ Laser Field," Journal of Physics B, Vol. 43, 2010, Article ID: 025203. http://dx.doi.org/10.1088/0953-4075/43/2/025203
[5] B. A. deHarak, L. Ladino, K. B. MacAdam and N. L. S. Martin, "High-Energy Electron-Helium Scattering in a Nd:YAG Laser Field," Physical Review A, Vol. 83, No. 2, 2011, Article ID: 022706. http://dx.doi.org/10.1103/PhysRevA.83.022706

[6] F. W. Byron Jr. and C. J. Joachain, "Electron-Atom Collisions in a Strong Laser Field," Journal of Physics B, Vol. 17, 1984, p. L295. http://dx.doi.org/10.1088/0022-3700/17/9/006

[7] A. Makhoute and D. Khalil, "The Effects of Laser Parameters on Electron-Atom Collisions," Progress in Laser and Electro-Optics Research, Nova Science Publishers, Inc., 2010.

[8] A. Makhoute and D. Khalil, "Phase Signatures in LaserAssisted Electron-Atom Collisions," European Physical Journal D, Vol. 46, No. 1, 2008, p. 77. http://dx.doi.org/10.1140/epjd/e2007-00280-8

[9] A. Makhoute, "Etude Théorique de Collisions ElectronsHelium en Présence de Champs Laser Intense," Ph.D. Dissertation, Universite Libre de Bruxelles, Brussels, 1991.

[10] D. V. Volkov, "Über eine Klasse von Lösungen der Diracschen Gleichung," Zeitschrift für Physik, Vol. 94, 1935, p. 50 .

[11] M. Dörr, C. J. Joachain and R. M. Potvliege, "Born-Floquet Theory of Laser-Assisted Electron-Atom Collisions," Physical Review A, Vol. 49, No. 6, 1994, p. 4852.

[12] G. Ferrante, C. Leone and F. Trombetta, "Laser Modifcation of the Exchange Scattering in Electron-Atom Collisions," Journal of Physics B, Vol. 15, 1982, p. L475. http://dx.doi.org/10.1088/0022-3700/15/13/011

[13] J. G. Story, D. I. Duncan and T. F. Gallagher, "Landau-Zener Treatment of Intensity-Tuned Multiphoton Resonances of Potassium," Physical Review A, Vol. 50, No. 2, 1994, p. 1607. http://dx.doi.org/10.1103/PhysRevA.50.1607

[14] C. J. Joachain, "Quantum Collision Theory," North-Holland, Amsterdam, 1983.

[15] P. Francken, Y. Attaourti and C. J. Joachain, "LaserAssisted Inelastic Electron-Atom Collisions," Physical Review A, Vol. 38, No. 4, 1988, p. 1785. http://dx.doi.org/10.1103/PhysRevA.38.1785

[16] D. Khalil, O. El Akramine, A. Makhoute, A. Maquet and R. Taïeb, "Light Polarization Eects in Laser-Assisted Elastic Electron-Helium Collisions: A Sturmian Approach," Journal of Physics B, Vol. 31, 1998, p. 1115. http://dx.doi.org/10.1088/0953-4075/31/5/018

[17] M. Bouzidi, A. Makhoute and M. N. Houkonounou, "Polarisation Effect of Laser Field in Inelastic Electron-Hydrogen Collisions," European Physical Journal D, Vol. 5, 2, 1999, p. 159. http://dx.doi.org/10.1007/s100530050239

[18] A. Dubois, A. Maquet and S. Jetzke, "ElectronH-Atom Collisions in the Presence of a Laser Field: One-Photon Free-Free Transitions," Physical Review A, Vol. 34, 1986, p. 1888 . 